\title{
A case of multiple myeloma presenting as scalp swelling with intracranial extension
}

\author{
Satya Bhusan Senapati, Sudhansu Sekhar Mishra, Manmath Kumar Dhir, Srikanta Das, Kalpalata Tripathy ${ }^{1}$ \\ Departments of Neurosurgery and ${ }^{1}$ Pathology, SCB Medical College and Hospital, Cuttack - 753 007, Odisha, India
}

\begin{abstract}
Multiple myeloma is a malignant neoplasm of bone marrow affecting plasma cells. It is usually detected in skull bone with characteristic features of multiple punched-out lesions. Its presentation as a solitary scalp swelling with underlying skull bone erosion and intracranial extension is very rare. A 35-year-old female presented to us with complains of rapidly growing left-side scalp swelling with right-side paresis and simple partial seizure of right upper limb. Local examination, X-ray skull, CT scan, and MRI of brain were suggestive of a malignant lesion. Near total excision of lesion was done. Histopathological study was suggestive of plasmacytoma of skull. Bone marrow study further confirmed it as a case of multiple myeloma. Cases presenting with solitary osteolytic skull lesions, possibility of plasmacytoma, or multiple myeloma should be kept in mind.
\end{abstract}

Key words: Multiple myeloma, osteolytic skull lesion, plasmacytoma

\section{Introduction}

In multiple myeloma, abnormal plasma cells accumulate in the bone marrow, where they interfere with the production of normal blood cells. Myeloma cells also produce paraprotein, an abnormal antibody which can cause kidney problems. A mnemonic commonly used to remember the common tetrad of multiple myeloma is $C R A B: C=$ Calcium (elevated), $\mathrm{R}=$ Renal failure, $\mathrm{A}=$ Anemia, $\mathrm{B}=$ Bone lesions. Bone pain, infection, renal failure, anemia, and neurological symptoms like headache, radicular pain, cord compression, and carpal tunnel syndrome are the common presenting symptoms.

\section{Case Report}

A 35-year-old female presented to us with complains of rapidly progressing scalp swelling over left side of

\begin{tabular}{|l|l|}
\hline \multicolumn{2}{|c|}{ Access this article online } \\
\hline Quick Response Code: & Website: \\
\hline & www.ruralneuropractice.com \\
\cline { 2 - 3 } & \\
\hline & DOI: \\
\hline
\end{tabular}

head for last 4 months, weakness of right hand followed by right lower limb for last 30 days, and simple partial seizure of right upper limb for last 15 days. She was anemic without lymphadenopathy or organomegally. We found a tense globular swelling over left frontal area of size 10-10-6 cm, with variegated consistency. This was fixed to underlying skull and skin. There was no visible impulse on cough, pulsation, or bruise over the swelling. There was an ulcer of about $2 \mathrm{~cm}$ in diameter situated over lateral aspect of swelling with signs of inflammation surrounding it [Figures 1a and b]. X-ray skull [Figure 1c], contrast enhanced computed tomography (CECT) of brain [Figure 2a], and contrast magnetic resonance imaging (MRI) of brain [Figures $2 \mathrm{~b}-\mathrm{d}$ ] were suggestive of an osteolytic skull lesion with intra cranial extension. Cerebral angio MRI showed a highly vascular tumor with patent superior longitudinal sinus [Figure 2e]. She was advised for preoperative tumor embolization, because of her financial constrain it was not carried out. Her laboratory examinations were within normal range except erythrosedimentation rate $(60 \mathrm{~mm} / \mathrm{h})$ and hemoglobin $8 \mathrm{~g} / \mathrm{dl}$. Work up for common metastatic lesions from breast, thyroid, kidney, and lungs were negative. Meningioma with malignant transformation, metastatic lesions, and plasmacytoma were kept as differential diagnosis.

An elliptical incision was given over the swelling taking ulcer in between the incision margins. We

Address for correspondence:

Dr. Satya Bhusan Senapati, Department of Neurosurgery, SCB Medical College and Hospital, Cuttack - 753 007, Odisha, India.

E-mail: satya.bhusan.senapati@gmail.com 
found a highly vascular, soft pinkish tumor eroding the skull bone and invading scalp layers [Figure 3a]. Most of the extra cranial portion was excised, because of severe bleeding patient went into shock. Patient revived after 4 units of blood transfusion. We went for second surgery, 7 days after first one. Dura margin was delineated by rongering out bone circumferentially around tumor margins. Tumor was found infiltrating dura and invading parenchyma [Figure 3b]. Dura excised along with adherent residual extracranial tumor followed by excision of invading intracranial tumor done [Figure 3c]. Duroplasty was done with synthetic dural substitute [Figure 3d]. Histopathological study of tumor sample came as plasmacytoma [Figures 4a and b]. On immunohistochemical staining, tumor cells were negative for CD-20 [Figure $4 \mathrm{~d}$ ] and positive

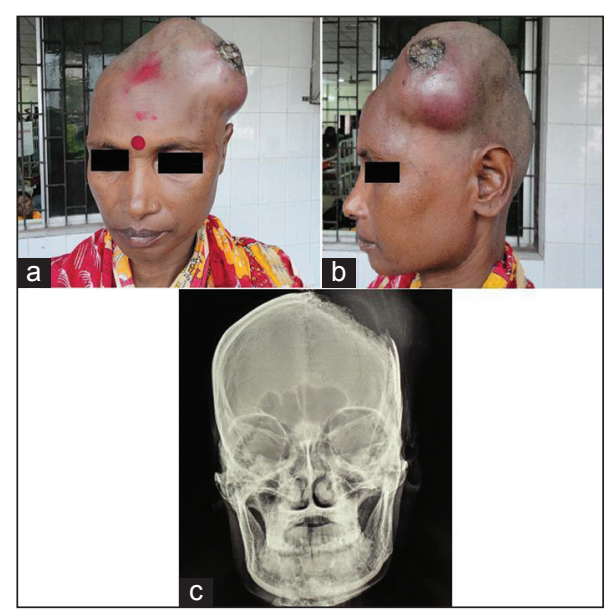

Figure 1: $(a, b)$ A tense globular swelling over left frontal area of size $10-10-6 \mathrm{~cm}$, with variegated consistency, fixed to underlying skull and skin. (c) X-ray skull showing large boney erosion in left fronto-parietal region with soft tissue swelling

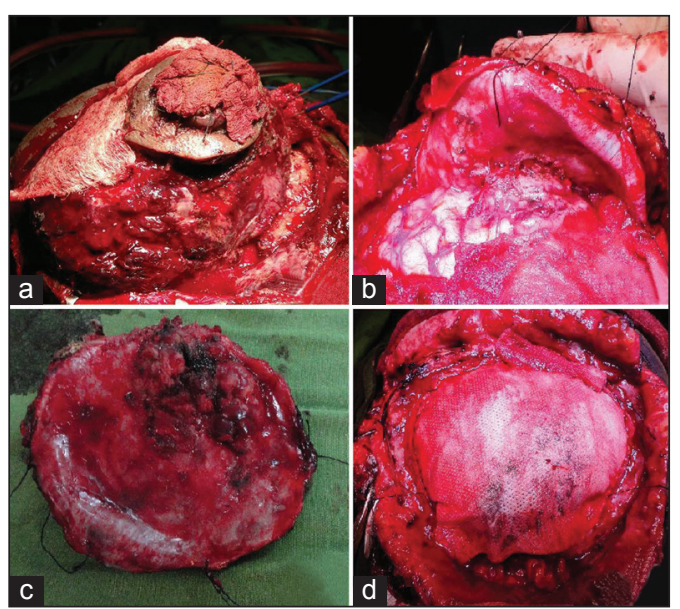

Figure 3: (a) A highly vascular, soft pinkish tumor eroding the skull bone, and invading scalp layers. (b) Dura reflected to show intraparenchymal infiltration of tumor. (c) Inner surface of excised dura showing tumor infiltration site. (d) Duroplasty done with synthetic dural substitute for CD-138. Bone marrow aspiration study showed increase in number of plasma cells, constituting 30\% of marrow nucleated cells. Both bi- and trinucleated plasma cells were seen [Figure 4c]. Serum protein electrophoresis showed monoclonal " $\mathrm{M}$ " spike and screening skeletal X-ray failed to found other lytic lesion.

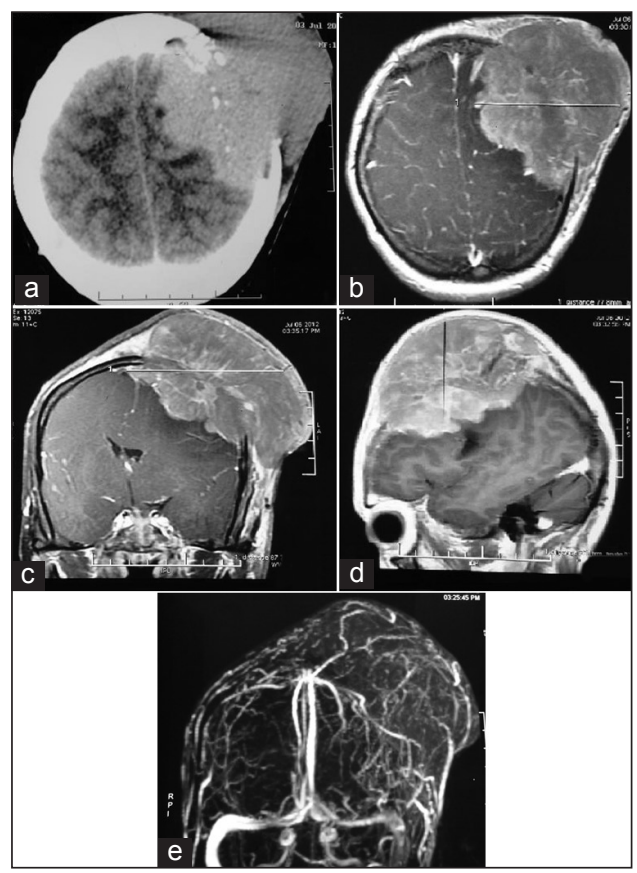

Figure 2: (a) CECT scan of brain showing a solid SOL in LT frontal lobe with erosion of LT frontal bone and involvement of scalp. (b-d) Contrast MRI of brain showing LT post superior-frontal extra axial lesion with signal characteristics of T1wi isointense, T2wi isointense to heterogeneous, large fairly marinated mass with homogenous enhancement and dural tail. (e) Cerebral angio MRI showing a highly vascular tumor with patent superior longitudinal sinus

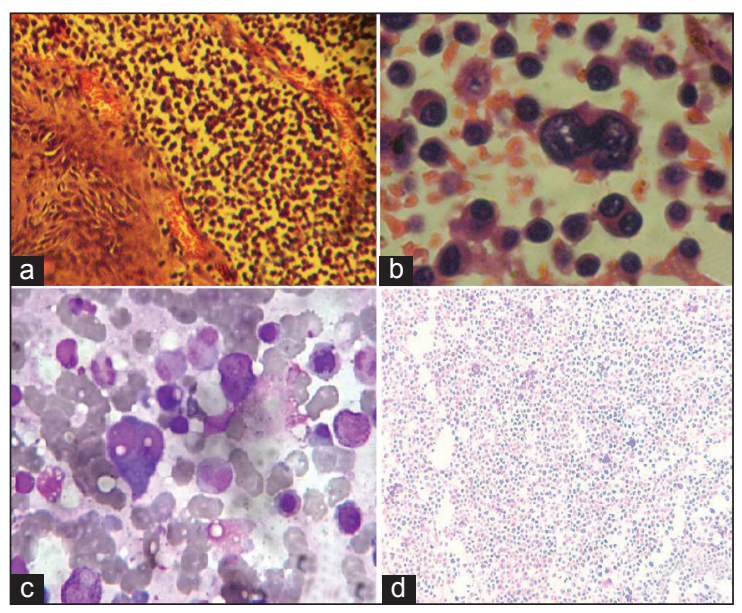

Figure 4: (a) Tumor specimen showing stratified squamous cell epithelium with subepithelium showing neoplastic cell $(20 \mathrm{X}-\mathrm{HandE}$ stain). (b) Tumor specimen showing binucleate and multinucleate form of plasma cell (40 X - HandE stain). (c) Bone marrow showing binucleate form of plasma cell. It constitutes $30 \%$ of marrow nucleated cells. (d) On immunohistochemical staining, tumor cells were negative for CD-20. 
Final diagnosis was multiple myeloma. Postoperatively, local radiotherapy (50 Gy) and chemotherapy (three cycles of melphalon plus dexamethasone) were given. At 3-month follow up, the patient is doing well with no local recurrence.

\section{Discussion}

Plasma cell neoplasms are divided into multiple myelomas, solitary plasmocytomas of bone, and extramedullary plasmocytomas. Multiple myelomas represent systemic disease, solitary plasmocytomas of bone, and extramedullary plasmocytomas represent local forms of plasma cell neoplasm. The International Myeloma Working Group had proposed new criteria for the diagnosis and classification of myeloma; they have broadly divided myeloma into symptomatic myeloma, asymptomatic myeloma, and monoclonal gammopathy of undetermined significance (MGUS). According to the criteria, symptomatic myeloma requires evidence of an M-protein in the serum and urine, bone marrow plasmacytosis, and related end-organ damage. The criteria for asymptomatic myeloma are M-protein levels $\geq 30 \mathrm{~g} / \mathrm{l}$ and/or bone marrow clonal cells $\geq 10 \%$, but no related organ or tissue impairment (ROTI). The criteria for MGUS are M-protein levels $<30 \mathrm{~g} / \mathrm{l}$ and/or bone marrow low clonal cells $<10 \%$ and no ROTI. Myelomarelated organ or tissue impairment (ROTI) adapted from International Myeloma Working Group, 2003, includes increased calcium levels, renal insufficiency, anemia, bone lesions, and other features like hyperviscosity, amyloidosis, and recurrent bacterial infections $(>2$ episodes in 12 months). ${ }^{[1,2]}$

The diagnosis of plasmacytoma is based on a radiologically solitary bone lesion, plasma cells in the biopsy specimen, fewer than 5\% plasma cells in bone marrow, less than $2.0 \mathrm{~g} / \mathrm{dl}$ monoclonal protein (M-protein) in the serum when present, negative urine test for Bence Jones protein, no evidence of hyperglobulinemia and hypercalcemia, and absence of anemia. ${ }^{[3-7]}$ Analyzing our case (bone lesion, M-protein in the serum, bone marrow plasmacytosis of $30 \%$, and related end-organ damage like anemia) supports the diagnosis as a case of symptomatic myeloma. Plasma cell neoplasm is radiosensitive; therefore, local debulking followed by radiotherapy had a good result on plasmacytoma. Once disease becomes multiple myeloma, then prognosis is not as good as plasmacytoma; chemotherapy should be added..$^{[8-11]}$ The potential for malignant systemic progression is higher for solitary plasmocytomas of bone than for extramedullary plasmocytomas. ${ }^{[12]}$ It has been reported that extramedullary localizations of myeloma originating from the cranial bone are usually responding well to new drugs (e.g., thalidomide, bortezomib, and lenalidomide) compared to CNS myeloma. ${ }^{[13]}$ It is always difficult to comment whether the brain tumor was the manifestation of multiple myeloma or the brain tumor of plasmacytoma had gradually transformed into multiple myeloma. In the absence of lytic lesions at other site, it would be appropriate to tail that our case is a plasmacytoma of skull bone that had transformed into multiple myeloma.

\section{Conclusion}

Cases presenting with solitary osteolytic skull lesions possibility of plasmacytoma or multiple myeloma should be kept in mind. Help of hematologist should be taken to differentiate plasmacytoma from multiple myeloma, as treatment modality differs from one another. Cases of plasmacytoma should be on lifelong regular follow up, as it has a tendency to progress into multiple myeloma. While debulking such big tumors help of interventional radiologist should be taken for tumor embolization, as it decrease blood loss intraoperatively.

\section{References}

1. International Myeloma Working Group: Criteria for the classification of monoclonal gammopathies, multiple myeloma and related disorders: A report of the International Myeloma Working Group. Br J Haematol 2003;121:749-57.

2. BCSH and UKMF Guidelines on the Management and Diagnosis of Multiple Myeloma Sept 2010. 14/10/2010.

3. Tanaka M, Shibui S, Nomura K, Nakanishi Y. Solitary plasmacytoma of the skull: A case report. Jpn J Clin Oncol 1998;28:626-30.

4. Naganuma H, Sakatsume S, Sugita M, Satoh E, Asahara T, Nukui H. Solitary plasmacytoma of the skull: Immunohistochemical study of angiogenic factors and syndecan-1 - two case reports. Neurol Med Chir (Tokyo) 2004;44:195-200.

5. Joshi A, Jiang D, Singh P, Moffat D. Skull base presentation of multiple myeloma. Ear Nose Throat J 2011;90:E6-9.

6. Caravita T, Siniscalchi A, Montinaro E, Bove R, Zaccagnini M, De Pascalis D, et al. Multiple myeloma and paget disease with abnormal skull lesions and intracranial hypertension. Mediterr J Hematol Infect Dis 2012;4:e2012068.

7. Taheri D, Suzangar H, Heidari F, Feshrakizadeh M, Suzangar M, Dolatkhah S. Skull Mass as the First Manifestation of Recurrent Multiple Myeloma in a Renal Transplant Patient. J Pak Med Assoc 2012;62:(3 Suppl 2)S76-8.

8. Engelsma RJ, De Bree R, Janssen JJ, Scheeren RA. Plasmacytoma of the mastoid bone: Solitary and systemic. J Laryngol Otol 2000;114:378-80.

9. Lorsbach RB, Hsi ED, Dogan A, Fend F. Plasma cell myeloma and related neoplasms. Am J Clin Pathol 2011;136:168-82.

10. Kyle RA, Therneau TM, Rajkumar SV, Larson DR, Plevak MF, Melton LJ $3^{\text {rd }}$. Incidence of multiple myeloma in Olmsted County, Minnesota: Trend over 6 decades. Cancer 2004;101:2667-74.

11. Ustuner Z, Basaran M, Kiris T, Bilgic B, Sencer S, Sakar B, et al. Skull base plasmacytoma in a patient with light chain myeloma. Skull Base 2003;13:167-71.

12. Reyhan M, Tercan F, Ergin M, Sukan, Aydin M, Yapar AF. Sonographic diagnosis of a tracheal extramedullary plasmacytoma. J Ultrasound Med 2005;24:1031-4. 
13. Gozzetti A, Cerase A, Lotti F, Rossi D, Palumbo A, Petrucci MT, et al. Extramedullary intracranial localization of multiple myeloma and treatment with novel agents: A retrospective survey of 50 patients. Cancer 2012;118:1574-84.
How to cite this article: Senapati SB, Mishra SS, Dhir MK, Das S, Tripathy $\mathrm{K}$. A case of multiple myeloma presenting as scalp swelling with intracranial extension. J Neurosci Rural Pract 2013;4:445-8.

Source of Support: Nil. Conflict of Interest: None declared. 